\title{
EVALUATION OF SINGLE LAYER POTENTIALS OVER CURVED SURFACES*
}

\author{
LEANDRO FARINA ${ }^{\dagger}$
}

\begin{abstract}
The evaluation of nearly singular single layer potentials encountered in boundary element methods is treated by a new approach. The potential is expressed as a sum of a onedimensional integral and a correction term that vanishes for planar surfaces. The small variance of the second term's integrand allows the use of a quasi-Monte Carlo quadrature. Numerical results show that a significant reduction in computational time is obtained over algorithms employing domain subdivisions.
\end{abstract}

Key words. numerical integration, potential theory, boundary integral method, Monte Carlo method

AMS subject classifications. 65D30, 65R20, 31C20, 65C05

PII. S1064827599363393

1. Introduction. In boundary element methods, the solution of a boundary value problem is expressed in terms of an integral over the boundary $S$ of the original domain. For potential problems, a solution $\phi$ is obtained by solving the Fredholm integral equation

$$
\lambda(p) \phi(p)+\int_{S} \phi(q) G(p, q) d \sigma=f(p), \quad p \in S,
$$

where $G=\frac{1}{4 \pi} \frac{1}{|p-q|} \cdot p=(\xi, \eta, \zeta) \in \mathbb{R}^{3}$ is called the field point.

Discretization of (1.1) poses the task of evaluating

$$
U=\int_{S} g(q) G(p, q) d \sigma_{q}
$$

called the single layer potential with density $g$. A number of methods has been described [4], [10] to deal with integrals where the integrand behaves like the fundamental solution of Laplace's equation. However, usually the assumption that $S$ is of a particular form or has a certain parametrization is made, and/or the case where the integral is nearly singular is ill treated. In this paper, we will relax the usual conditions over the parametrization on $S$, and we also treat the case where $U$ assumes a moderate to a highly near singular character, as explained below. This is a topic of interest in the boundary element method, in particular, in higher-order methods.

Let $S$ be defined by a mapping $T: \mathcal{P}=[a, b] \times[c, d] \longrightarrow S$ with the property

$$
T(\partial \mathcal{P})=\partial S
$$

* Received by the editors November 5, 1999; accepted for publication (in revised form) November 27, 2000; published electronically June 5, 2001. This work was supported by the Industry Consortium Numerical Analysis for Wave Loads on Offshore Structures, Cambridge, MA, 1997-1999, Mobil, Statoil, DNV Software, Shell, OTRC, Petrobras, NorskHydro, Exxon, Chevron, SAGA, NSWC.

http://www.siam.org/journals/sisc/23-1/36339.html

${ }^{\dagger}$ Research Laboratory of Electronics, MIT, Cambridge, MA 02139. Current address: Centro de Previsão de Tempo e Estudos Climáticos (CPTEC), Instituto Nacional de Pesquisas Espaciais (INPE), Cachoeira Paulista, SP 12630-000, Brazil (farina@cptec.inpe.br). 
It is useful to classify $U$ according with the order of magnitude of the distance $\operatorname{dist}(p, S)$ between the field point $p$ and the surface $\mathrm{S}$. Thus we say

$$
\begin{aligned}
\text { if } \operatorname{dist}(p, S)=O(1), & U \text { is regular, } \\
\text { if } 0<\operatorname{dist}(p, S) \leq o(1), & U \text { is nearly singular, and } \\
\text { if } \operatorname{dist}(p, S)=0, & U \text { is singular. }
\end{aligned}
$$

We are mainly interested in the case in (1.5). In this case, the integral $U$ above is regular. However, as the integrand is near a singularity, standard quadrature formulae are not appropriate. This case appears in the boundary element method, typically in applications involving bodies with thin components where one part of $S$ is close to another part of this surface. Moreover, the occurrence of nearly singular integrals is also associated with more general types of domains, depending on how the surface is discretized or panelized. A large difference in size between two panels may create the condition described in (1.5). Recently, it was shown by Luo, Liu, and Berger [7] that conventional boundary integral methods will not degenerate even when applied to thin structures with the thickness to length ratio in the micro $\left(10^{-6}\right)$ or nano $\left(10^{-9}\right)$ scales. This is true as long as numerical difficulties, such as the calculation of the nearly singular integrals, are addressed.

2. Semianalytical approach. Integrals of type (1.2), where $g$ is a polynomial, can be evaluated in closed form when the surface $S$ is a flat polygon (see Newman [8]). However, for an arbitrary surface $S$, numerical integration becomes mandatory in the evaluation of $U$. Our approach here is to use analytical evaluation to a maximum degree in this ultimately numerical task. This will sustain the accuracy and efficiency of an analytical evaluation into the method.

Thus we decompose the surface integral $U$ as

$$
U=U_{o}+U_{c}
$$

where $U_{o}$ is a planar approximation to $U$ in the sense that it is given as an integral over a flat domain in $\mathbb{R}^{3}$ and it coincides with $U$ when $S$ is flat. $U_{c}$ is the correction due to this approximation. As we will see, the inner integral in $U_{o}$ will be evaluated analytically.

A precise expression for $U_{o}$ and $U_{c}$ will be given later. Let us first describe the change of variables defining the auxiliary flat domain.

3. New coordinate systems. The function $G$ presents a weak singularity at $q=(x, y, z)=p$, where it is unbounded. The fact that this function's integral is finite can be easily proved by using polar coordinates, since the jacobian will cancel the singularity. This also suggests a way of evaluating $U$ numerically as a regular integral. Consider a new coordinate system given by

$$
(\tilde{x}, \tilde{y}, \tilde{z})=M^{-1}\left(\begin{array}{l}
x \\
y \\
z
\end{array}\right),
$$

where the field point $p$ is the origin lying on the $\tilde{x} \tilde{y}$-plane, denoted by $D . M^{-1}$ is a $3 \times 3$ matrix. Using the polar coordinates $\rho=\sqrt{\tilde{x}^{2}+\tilde{y}^{2}}, \theta=\arctan (\tilde{y} / \tilde{x})$ gives

$$
U=\frac{1}{4 \pi} \int_{0}^{2 \pi} \int_{0}^{\mathcal{R}(\theta)} g(\rho, \theta) \frac{\rho}{\sqrt{\rho^{2}+h^{2}}} \tilde{J} d \rho d \theta
$$




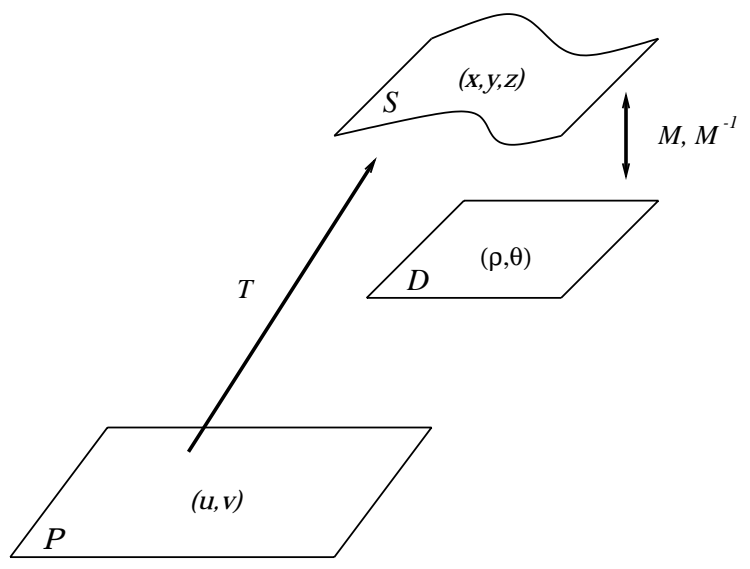

FIG. 1. The domains $\mathcal{P}, S, D$ and the transformations $T, M$, and $M^{-1}$.

where $h=\tilde{z}(\rho, \theta)$ and $\mathcal{R}(\theta)$ is the value of $\rho$, as a function of $\theta$, at the boundary of $S$. $\tilde{J}$ is the jacobian of the transformation $M^{-1}$, and it is given by $\tilde{J}=\frac{1}{n_{D} \cdot n_{S}}$, where $n_{D}$ and $n_{S}$ are the respective normal vectors to $D$ and $S$ at $q$. Because $D$ is flat, $\tilde{J}=n 3$, where $n_{3}$ is the third component of $n_{S}$.

With this new setting, we see that as $q$ approaches $p$, the integrand remains bounded and approaches the value of $g \tilde{J}$ at that point. Thus, in our approach, the integral defining $U$ is represented in the physical three-dimensional space.

There are three domains of interest in our problem: the parameter domain

$$
\mathcal{P}=[a, b] \times[c, d],
$$

the three-dimensional space $\mathbb{R}^{3}$ (where $S$ is embedded), spanned by the canonical basis

$$
\mathcal{C}=\{x, y, z\}
$$

or by the alternative basis

$$
\mathcal{B}=\{\tilde{x}, \tilde{y}, \tilde{z}\}
$$

and the flat domain

$$
D=\operatorname{span}\{\tilde{x}, \tilde{y}\}=\operatorname{span}\{\rho, \theta\}
$$

where the integrand is regularized.

The main difficulty associated with evaluating (3.1) is that it is not possible, in general, to evaluate $\mathcal{R}, h$, and $\tilde{J}$ as functions of $(\rho, \theta)$. This is a result of the lack of restriction on $S$. Indeed, we assume $S$ can be any parametrized surface with the property (1.3). Then, the fact that the transformation $T: \mathcal{P}=[a, b] \times[c, d] \longrightarrow S$ is not, in general, invertible prevents us from evaluating $\mathcal{R}, h$, and $\tilde{J}$ directly as functions of variables in $S$ or $D$ (see Figure 1) and therefore from using a quadrature formula where the location of the integration points are predefined. In other words, the control over the integration points is lost; the relative locations of these points in $\mathcal{P}$ will be altered by the transformation $T$. Thus our approach is to use a interpolation quadrature [2] based on linear functions or on splines for computing $U_{o}$ and a Monte Carlo method for $U_{c}$. We will describe and give details about this approach in the following sections. 


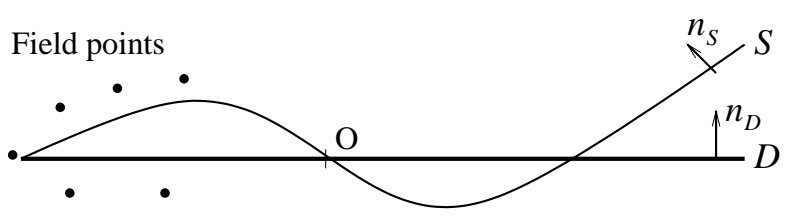

FIG. 2. The domain $D$ and the surface $S$.

4. Independence on the field point. We saw that the change of variables described in the last section, above formula (3.1), provides a regular and simple integrand. However, the new variables are dependent on the field point $p$. This feature is inconvenient from the computational point of view if the objective is to evaluate $U$ for several, say, $L$ field points, as is usual in boundary element methods for numerical solution of integral equations. Therefore, we will use a similar change of variables, but independent on the field point. In this way, the matrix of the linear transformation between $\mathcal{C}$ and $\mathcal{B}$ is determined once for all computations associated with the surface $S$. Moreover, the integration points and certain parts of the integrands are computed only once. Then consider $\mathcal{B}=\{\tilde{x}, \tilde{y}, \tilde{z}\}$, as defined in section 3 , but with a fixed origin $O=\left(O_{1}, O_{2}, O_{3}\right) \in S$, which will be a free parameter specified as one finds appropriate (see Figure 2). The optimum location of $O$ is found by minimizing

$$
\max \{|\tilde{z}|:((\tilde{x}, \tilde{y}, \tilde{z})-O) \in S\} .
$$

Let us assume $g$ may be expanded in powers of $x-\xi, y-\eta$, and $z-\zeta$. In view of this, for the evaluation of $U$, it is sufficient to consider $U^{\mu \nu v}:=\int_{S} P_{\mu \nu v}(p-q) G(p, q) d \sigma_{q}$, where $P_{\mu \nu v}(p-q)=(x-\xi)^{\mu}(y-\eta)^{n} u(z-\zeta)^{v}$.

Now define $U_{o}^{\mu \nu v}$ as

$$
U_{o}^{\mu \nu v}=\frac{1}{4 \pi} \int_{0}^{2 \pi} \int_{0}^{\mathcal{R}(\theta)} P_{\mu \nu v}(p-q) \frac{\rho}{\sqrt{\rho^{2}+b \rho+a}} d \rho d \theta,
$$

where $a=\xi^{2}+\eta^{2}+(\bar{z}-\zeta)^{2}$ and $b(\theta)=-2(\xi \cos \theta+\eta \sin \theta)$. The value $\bar{z}$ represents an average value of $\tilde{z}$ and can be taken as $\bar{z}=O_{3}$.

We now have

$$
U^{\mu \nu v}=U_{o}^{\mu \nu v}+U_{c}^{\mu \nu v}
$$

where

$$
U_{c}^{\mu \nu v}=\frac{1}{4 \pi} \int_{0}^{2 \pi} \int_{0}^{\mathcal{R}(\theta)} P_{\mu \nu v}(p-q)\left\{\frac{\rho}{r} \frac{1}{n 3}-\frac{\rho}{\bar{r}}\right\} d \rho d \theta
$$

with $r=|p-q|$ and $\bar{r}=\sqrt{(\tilde{x}-\xi)^{2}+(\tilde{y}-\eta)^{2}+(\bar{z}-\zeta)^{2}}=\sqrt{\rho^{2}+b \rho+a}$.

5. Quadrature of $\boldsymbol{U}_{\boldsymbol{o}}$. The objective in this section is to express (4.1) in the form

$$
U_{o}^{n m}=\frac{1}{4 \pi} \int_{0}^{2 \pi} F(\mathcal{R}(\theta), \theta) d \theta
$$

where $F$ is an exact closed form expression for the inner integral in (4.1), and to subsequently apply a one-dimensional quadrature to (5.1). This quadrature will be 
based on nodes $\left(\theta_{i}, F_{i}\right)$ obtained from a sample of points $\left(u_{i}, v_{i}\right)$ selected in $\partial \mathcal{P}:=$ $\mathcal{P}_{1} \cup \mathcal{P}_{2} \cup \mathcal{P}_{3} \cup \mathcal{P}_{4}$, where

$$
\begin{array}{ll}
\mathcal{P}_{1}=\{(u, v): v=c\}, & \mathcal{P}_{2}=\{(u, v): u=b\}, \\
\mathcal{P}_{3}=\{(u, v): v=d\}, & \mathcal{P}_{4}=\{(u, v): u=a\} .
\end{array}
$$

Condition (1.3) assures that $\mathcal{R}$ is in fact evaluated at the boundary of $S$. This is the only place where $(1.3)$ is used. Thus the quadrature nodes $\left(\theta_{i}, F_{i}\right)$ are obtained without the knowledge of their exact location. For smooth integrands $F$, a quadrature based on interpolating cubic splines or Hermite functions [5] can be used. Alternatively, noninterpolating methods could be used, such as the locally corrected quadrature proposed by Strain [9], where singularities are allowed in the integrand $F$.

In order to make $F(\mathcal{R}(\theta), \theta)$ explicit, note that

$$
(x, y, z)=M\left(\begin{array}{c}
\tilde{x} \\
\tilde{y} \\
\tilde{z}
\end{array}\right)-O,
$$

where $M=\left\{M_{i j}\right\}$ is a $3 \times 3$ matrix. It follows that on $D$ we have

$$
\left\{\begin{array}{l}
x=M_{11} \rho \cos \theta+M_{12} \rho \sin \theta-O_{1}, \\
y=M_{21} \rho \cos \theta+M_{22} \rho \sin \theta-O_{2}, \\
z=M_{31} \rho \cos \theta+M_{32} \rho \sin \theta-O_{3} .
\end{array}\right.
$$

Therefore,

$$
F(\mathcal{R}(\theta), \theta)=\int_{0}^{\mathcal{R}(\theta)}(c \rho+d)^{\mu}(e \rho+f)^{\nu}(h \rho+l)^{v} \frac{\rho}{\sqrt{\rho^{2}+b \rho+a}} d \rho
$$

where $d=-\xi-O_{1}, f=-\eta-O_{2}, l=-\zeta-O_{3}, c(\theta)=M_{11} \cos \theta+M_{12} \sin \theta, e(\theta)=$ $M_{21} \cos \theta+M_{22} \sin \theta$, and $h(\theta)=M_{31} \cos \theta+M_{32} \sin \theta$. Expanding the binomials in (5.2), we get

$$
\begin{aligned}
F(\mathcal{R}(\theta), \theta) & =\sum_{i=0}^{\mu} \sum_{j=0}^{\nu} \sum_{k=0}^{v}\left(\begin{array}{c}
\mu \nu v \\
i j k
\end{array}\right) d^{i} f^{j} l^{k} c^{\mu-i}(\theta) e^{\nu-j}(\theta) h^{v-k}(\theta) \\
& \times H_{\mu+\nu+v-i-j-k+1}(\theta),
\end{aligned}
$$

where $\left(\begin{array}{c}\mu \nu v \\ i j k\end{array}\right):=\left(\begin{array}{c}\mu \\ i\end{array}\right)\left(\begin{array}{c}\nu \\ j\end{array}\right)\left(\begin{array}{l}v \\ k\end{array}\right)$ and

$$
H_{t}(\theta)=\int_{0}^{\mathcal{R}(\theta)} \frac{\rho^{t}}{\sqrt{\rho^{2}+b \rho+a}} d \rho
$$

The integral (5.3) can be recursively evaluated using [3]

$$
H_{t}(\theta)=\frac{\rho^{t-1}}{t} \sqrt{\rho^{2}+b \rho+a}-\frac{(2 t-1) b}{2 t} H_{t-1}(\theta)-\frac{(t-1) a}{t} H_{t-2}(\theta),
$$


with the starting functions

$$
\begin{aligned}
& H_{0}(\theta)= \begin{cases}\left.\log \left|2 \rho+b+2 \sqrt{\rho^{2}+b \rho+a}\right|\right|_{\rho=0} ^{\rho=\mathcal{R}(\theta)}, & 4 a \neq b^{2}, \\
\left.\log |b+2 \rho|\right|_{\rho=0} ^{\rho=\mathcal{R}(\theta)}, & 4 a=b^{2}\end{cases} \\
& H_{1}(\theta)=\left.\sqrt{\rho^{2}+b \rho+a}\right|_{\rho=0} ^{\rho=\mathcal{R}(\theta)}-\frac{b}{2} H_{0}(\theta) .
\end{aligned}
$$

6. Quadrature of $\boldsymbol{U}_{\boldsymbol{c}}$ by quasi-Monte Carlo. The evaluation of expression (4.2) is more complex than the numerical integration of (4.1). In the case of $U_{o}$, it was possible to separate the variables $\rho$ and $\theta$ in order to evaluate the inner integral analytically. Because $n 3$ is an arbitrary function associated with the given surface $S$, the correction component $U_{c}$ does not permit a similar variable separation. Thus $U_{c}$ has to be integrated numerically as a two-dimensional integral. Since $U_{c}$ presents the same discretization condition as $U_{o}$, namely, the location of the nodes in the $(\rho, \theta)$ variables is arbitrary, this makes the problem more delicate. The apparent solution ${ }^{1}$ seems to use interpolation in two variables, imitating the procedure for evaluating (5.1). However, we believe that interpolation provides neither more efficiency nor more accuracy than a Monte Carlo quadrature. Traditionally, since its convergence rate is independent of the problem dimension, Monte Carlo quadrature has been used in high-dimensional integrals as an efficient and robust alternative to grid-based methods. In what follows we will outline the reasons for using a Monte Carlo type of quadrature in a low-dimensional (i.e., two-dimensional) integral and describe the specific approach employed.

Let $\left(\rho_{i}, \theta_{i}\right)_{i=\{1, \ldots, N\}}$ be a sequence of points in $D$. To evaluate (4.2) we use an integration formula $Q_{N}$ of the form

$$
Q_{N}\left(U_{c}\right)=A(D) \mu_{N}(K)
$$

where

$$
\begin{aligned}
A(D) & =\frac{1}{4 \pi} \int_{0}^{2 \pi} \mathcal{R}(\theta) d \theta, \\
K & =P_{\mu \nu v}(p-q)\left\{\frac{\rho}{r} \frac{1}{n 3}-\frac{\rho}{\bar{r}}\right\},
\end{aligned}
$$

and $\mu_{N}$ denotes the sample mean of $K$ on $D$, given as

$$
\mu_{N}(K)=\frac{1}{N} \sum_{i=1}^{N} K\left(\rho_{i}, \theta_{i}\right) .
$$

Note that the evaluation of $A(D)$ is intimately related with the numerical integration of $U_{o}$. From (5.1), we see that $A(D)$ is a special case, where $F(\mathcal{R}(\theta), \theta) \equiv \mathcal{R}(\theta)$.

\footnotetext{
${ }^{1}$ Strain's approach [9] does not seem applicable here because of its restriction that the domain of integration must be a hypercube.
} 


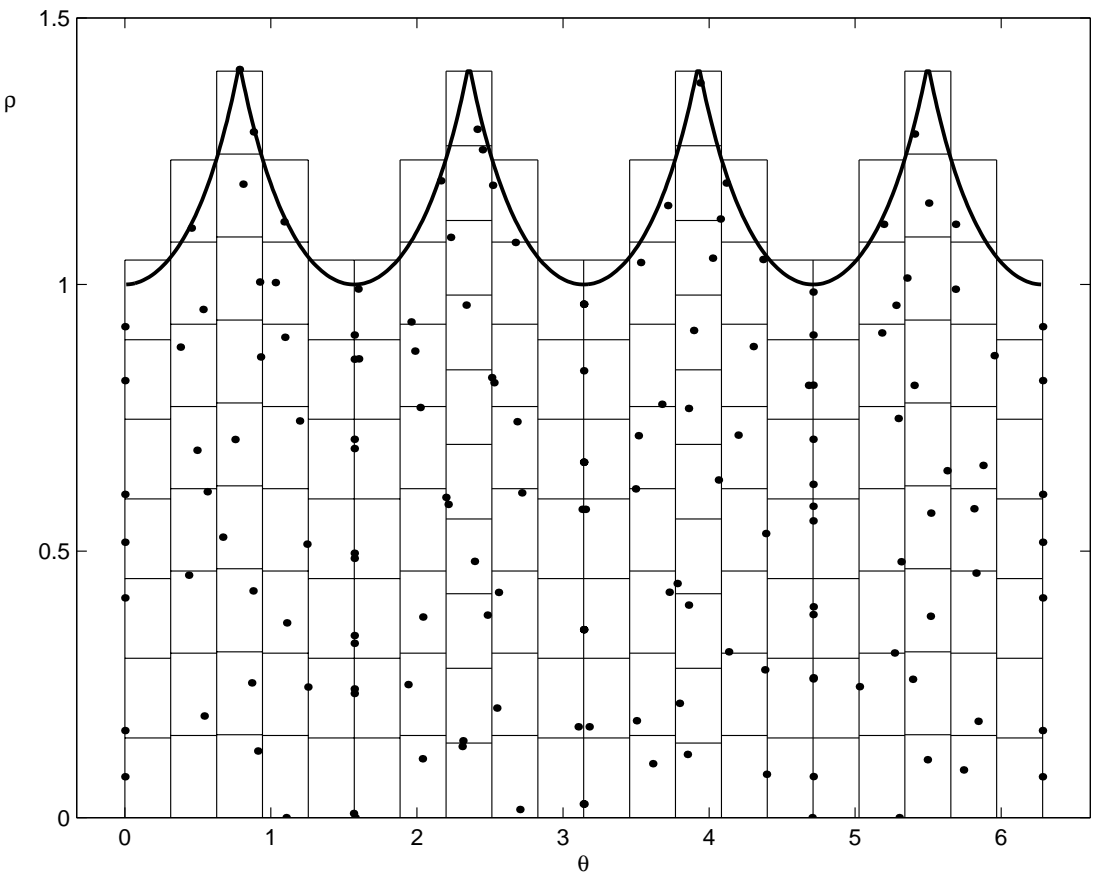

FIG. 3. The effect of the partitioning algorithm represented by the location of the integration nodes in the $\theta-\rho$ plane bounded by $\mathcal{R}(\theta)$. Here $N_{c}=1$, and $S$ is a curved quadrilateral.

As is well known in the theory of Monte Carlo methods, there are basically two resources for improving the convergence rate of our approximation (6.1) to $U_{c}$. One is to force the nodes to be as uniform as possible, and the other is to reduce somehow the variance

$$
\sigma^{2}(K):=\int_{D}(K-\bar{K})^{2} d \rho d \theta,
$$

where $\bar{K}$ is the mean of the integrand $K$.

We will use both means to obtain a suitable algorithm for the computation of $U_{c}$. The particular procedure to achieve this goal in the correction integral will be described next.

The control variates [1], [6, Chapter 6] form of variance reduction is in fact used from the beginning in our approach to evaluate the single layer potential as in the decomposition (2.1). The term

$$
\left\{\frac{\rho}{r} \frac{1}{n 3}-\frac{\rho}{\bar{r}}\right\}
$$

in $K$ vanishes where the $S$ is flat and, for moderate curvatures in $S$, presents small variances.

The other resource that we will use to improve convergence rates is the uniformization of the nodes distribution over the domain of integration.

The sequence of the nodes will fill the integration domain more uniformly and less uncorrelatedly than random nodes characterizing a quasi-Monte Carlo formula. For 


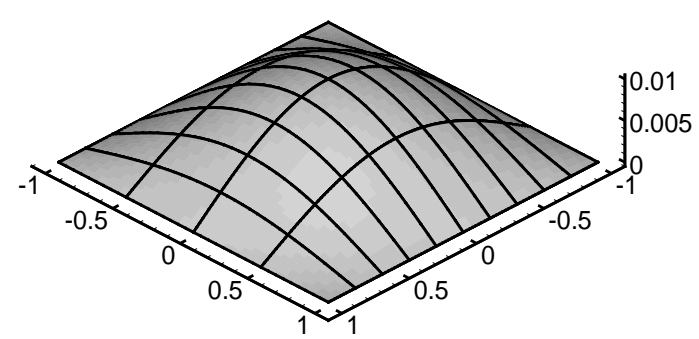

FIG. 4. The surface $S_{c}$.

our case, the transformations $T$ and $M$ would void any deterministic choice of points in $\mathcal{P}$. In order to obtain low-discrepancy nodes, we combine the stratification and the acceptance-rejection methods [6] into a partitioning algorithm. Thus we divide the $\rho-\theta$ domain in approximately equal sized cells, or subregions, and we accept or reject the pseudorandom generated points for the formula (6.1) until an equal number of subnodes $N_{c}$ are present in each cell. A typical example of the effect of this algorithm can be seen in Figure 3, where the distribution of the integration nodes in the $\theta-\rho$ plane is represented. In this figure, the bounding curve is $\mathcal{R}(\theta)$, given for a curved quadrilateral with equal, straight sides, and the cells are rectangles with one subnode.

7. Numerical results. In this section we present numerical results obtained from the Monte Carlo-based method described above and compare them with a standard quadrature employing the Gauss-Legendre formula combined with subdivision of the integration domain. This subdivision takes place whenever $\operatorname{dist}(p, S)$ becomes small compared with the area of $S$. All results in this section are believed to have relative error less than $0.3 \%$.

Let $S_{c}$ be a curved quadrilateral given by

$$
S_{c}:(x(u, v), y(u, v), z(u, v))=T(u, v)=(u, v, 0.01 \sin u \sin v+0.01)
$$

and represented in Figure 4. In Figure 5, the dotted line represents a subdivision method, and the solid line indicates quasi-Monte Carlo. The computing time (on a UNIX workstation) is plotted as a function of the number $L$ of single layer potentials computed, each layer potential corresponding to a field point. Thus up to 1000 field points $p_{i}$ were chosen satisfying the condition $\operatorname{dist}\left(p_{i}, S\right) \geq 10^{-2}$. For $L \approx 28$, quasiMonte Carlo requires less computational time than a standard subdivision method. The graph shows monotonically increasing linear functions, and for a large number of evaluations (1000 field points), we see a factor of 3 difference between the two methods. This difference is due to the fact that in our field point independent approach, information such as the location of the integration nodes and parts of the integrands are reused in all the integrals evaluated. In particular, calls to a subroutine or function defining the transformation $T$ are made only for the first integral. Furthermore, the value of $A(D)$ in (6.1) is fixed. Hence the effort to evaluate successive integrals becomes minimal with the increase of $L$.

Let us now comment on another example. Suppose now that the surfaces over which the integration is done are a family of planar quadrilaterals given as

$$
S_{\epsilon}:(x(u, v), y(u, v), z(u, v))=T(u, v)=(u, v, \epsilon),
$$

where $10^{-7} \leq \epsilon \leq 0.1$. In this case, $U_{c}=0$, and the Monte Carlo method is not 


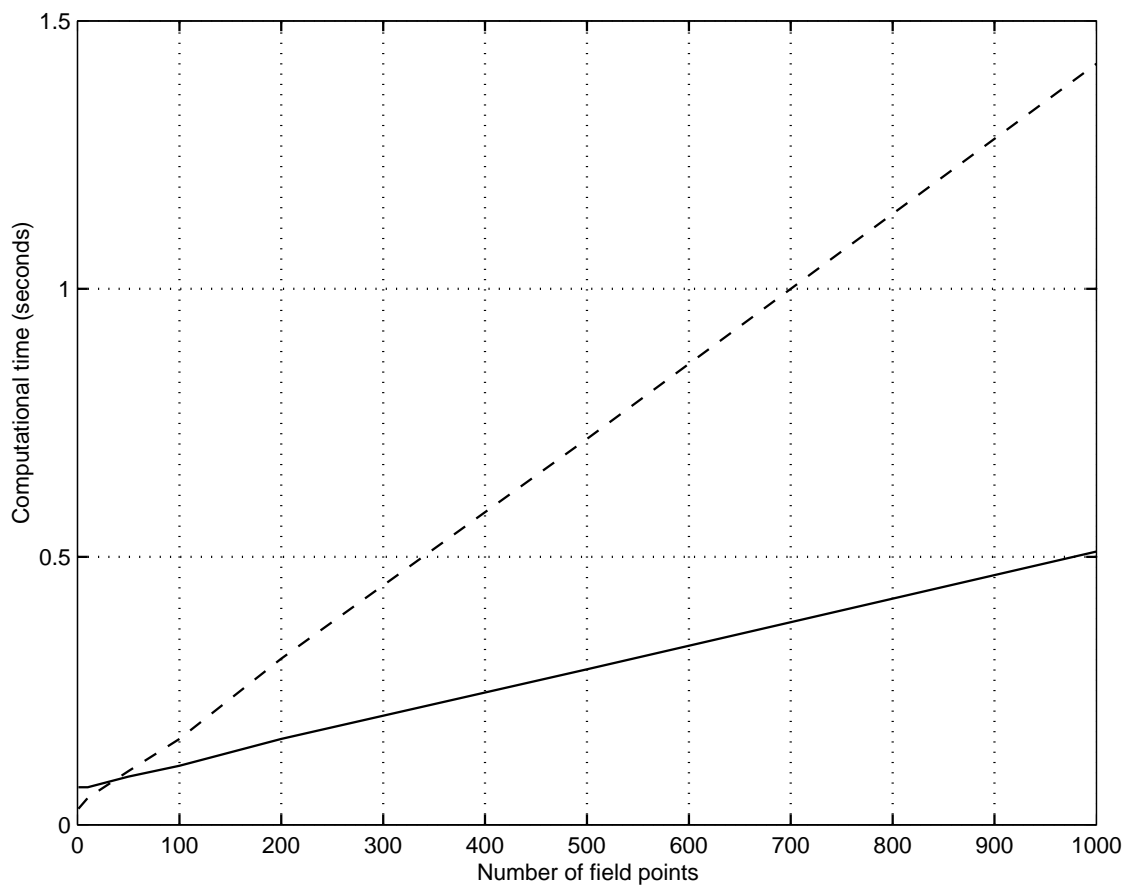

FIG. 5. The results for the surface $S_{c}$ and for $\operatorname{dist}\left(p_{i}, S\right) \geq 10^{-2}$. The dotted line represents the subdivision method, and the solid line indicates the quasi-Monte Carlo method.

necessary to use. The integrals are evaluated by one-dimensional quadrature since

$$
U=U_{o} .
$$

In Figure 6, with the same representations for the dotted and solid line, we see the computational time as a function of $\operatorname{dist}\left(p_{i}, s\right)=\epsilon$, in the range of $\epsilon$ specified above. Here $L=500$, and the field points are distributed uniformly over the $x$-axis. As can be seen, the computation of $U_{o}$ is not affected by the degree of the near singularity, represented by $\epsilon$. On the other hand, the use of standard quadratures, even with domain subdivision, suffers from the necessity of an excessive number of nodes and/or domain subdivisions.

It should be noted that under certain conditions the algorithm presented here will not be appropriate in its present form. Although it will provide good results for the case (1.4) because the integrand will be smooth and well approximated by polynomials, its efficiency will not be superior to a regular quadrature with a small number of nodes. Also, for surfaces with large curvature, the contribution from $U_{c}$ will be large compared with $U_{o}$, and a large number of nodes may be required to sustain accuracy.

8. Conclusion. A method for computing nearly singular single layer potentials has been introduced. Restrictions on the parametrization of the integration domain are relaxed, and the original integral is decomposed in a sum two terms, $U_{o}$ and $U_{c}$, where the first is an approximation which coincides with the single layer potential when the surface is planar. The term $U_{c}$ provides the correction when the surface loses its planar character. This decomposition not only allows $U_{o}$ to be evaluated in terms 


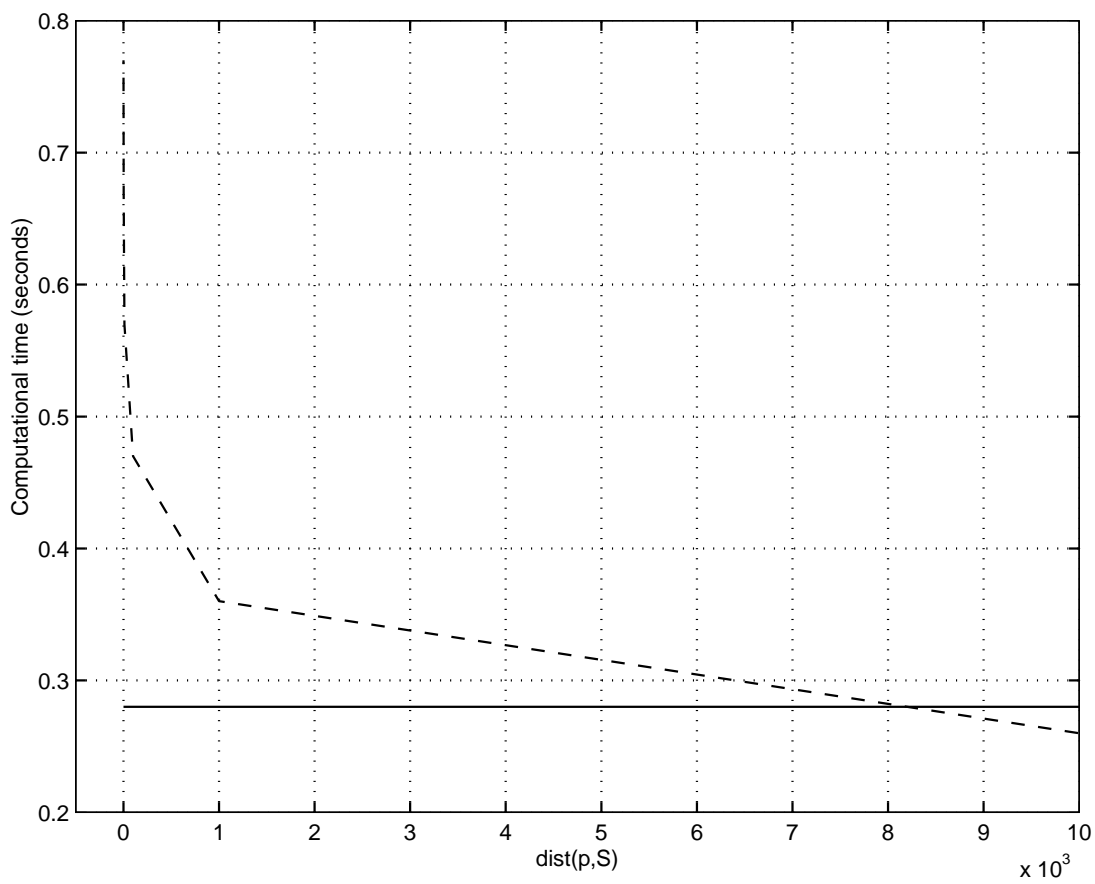

FIG. 6. The results for $S_{\epsilon}$ and for $L=500$. The dotted line represents the subdivision method, and the solid line indicates the quasi-Monte Carlo method.

of a one-dimensional regular quadrature but also reduces the integrand variance in $U_{c}$, making it viable to apply a two-dimensional quadrature based on pseudorandom nodes. This latter quadrature also employs a uniformization algorithm to the nodes distribution in order to improve the formula convergence rate. Also, because the quadrature of $U_{c}$ is not grid-based, adaptiveness can be easily incorporated.

Usually, in boundary element methods, integrals like $U$ have to be computed several times according with different field points. The method described above explores this fact by reusing information from previous computations. Numerical results clearly show an economy in computational costs for this approach when compared with standard quadrature employing domain subdivisions. For surfaces with moderate curvature, previously prohibitive nearly singular integrals where the ratio distance from the field point to the surface average length reaches $10^{-7}$ or less can be dealt with using the present algorithm without significant increase in the computational cost.

It may be possible to extend this approach to other types of integrands, such as the double layer potential. In order to achieve this successfully, one needs to efficiently integrate the strong near singularity that will be present in $U$.

Acknowledgments. The author thanks the people at the Computational Hydrodynamics Facility at MIT for helpful discussions about this work.

\section{REFERENCES}

[1] R. E. Caflisch, Monte Carlo and quasi-Monte Carlo methods, in Acta Numerica, 1998, Acta Numer. 7, Cambridge University Press, Cambridge, UK, 1998, pp. 1-49. 
[2] P. J. Davis and P. Rabinowitz, Methods of Numerical Integration, 2nd ed., Academic Press, Orlando, FL, 1984.

[3] I. S. Gradshteyn and I. M. Ryshik, Table of Integrals, Series, and Products, 5th ed., Academic Press, Boston, 1994.

[4] W. Hackbusch and S. A. Sauter, On numerical cubatures of nearly singular surface integrals arising in BEM collocation, Computing, 52 (1994), pp. 139-159.

[5] D. Kahaner, C. B. Moler, And S. NAsh, Numerical Methods and Software, Prentice Hall, Englewood Cliffs, NJ, 1989.

[6] A. R. Krommer and C. W. Ueberhuber, Computational Integration, SIAM, Philadelphia, 1998.

[7] J. Luo, Y. Liu, And E. Berger, Analysis of two-dimensional thin structures (from micro- to nano-scales) using the boundary element method, Comput. Mech., 22 (1998), pp. 404-412.

[8] J. N. Newman, Distributions of sources and normal dipoles over a quadrilateral panel, J. Engrg. Math., 20 (1986), pp. 113-126.

[9] J. StRAin, Locally corrected multidimensional quadrature rules for singular functions, SIAM J. Sci. Comput., 16 (1995), pp. 992-1017.

[10] J. C. F. TELLES, A self-adaptive co-ordinate transformation for efficient numerical evaluation of general boundary element integrals, Internat. J. Numer. Methods Engrg., 24 (1987), pp. 959-973. 